\title{
openheart Comprehensive update on the new indications for transcatheter aortic valve replacement in the latest 2017 European guidelines for the management of valvular heart disease
}

\author{
Tasalak Thonghong, Ole De Backer, Lars Søndergaard
}

To cite: Thonghong T, De Backer 0, Søndergaard L. Comprehensive update on the new indications for transcatheter aortic valve replacement in the latest 2017 European guidelines for the management of valvular heart disease. Open Heart 2018;5:e000753. doi:10.1136/ openhrt-2017-000753

Received 29 November 2017 Accepted 6 February 2018

Check for updates

Cardiology, Rigshospitalet, Copenhagen, Denmark

Correspondence to Dr Ole De Backer; ole. debacker@gmail.com

\section{ABSTRACT}

New European guidelines on the management of valvular heart disease - supported by the European Society of Cardiology (ESC) and European Association of CardioThoracic Surgery (EACTS) — were recently published. Although these guidelines are very comprehensive, these typically are not very inviting to read. In this document, we aimed to distil all the information about transcatheter aortic valve replacement (TAVR) in the new 2017 ESC/EACTS guidelines to the essential and give additional comments on the position of TAVR in 2017.

\section{INTRODUCTION}

Aortic valve stenosis is the most common primary valve disease leading to surgical or transcatheter valve replacement in Europe and North America, with a growing prevalence due to the ageing population. ${ }^{12}$ Driven by technological developments, the treatment of patients with severe aortic stenosis has seen a significant change over the past decade. $^{3}$

Since the 2012 European Society of Cardiology (ESC) /European Association of CardioThoracic Surgery (EACTS) guidelines on the management of valvular heart disease, ${ }^{4}$ a large amount of new data have accumulated, particularly in the field of catheter-based treatment of aortic valve stenosis. There have been five randomised clinical trials (RCTs) comparing surgical aortic valve replacement (SAVR) and transcatheter aortic valve replacement (TAVR) as well as large-scale registry data. ${ }^{5-9}$ Indications for TAVR have expanded as there is new evidence for the lower risk populations. ${ }^{7-11}$ This has resulted in an exponential growth in the number of TAVR procedures performed in Europe and North America, with the number of TAVR now surpassing the number of isolated SAVR in several European countries. Surprisingly, the number of SAVR procedures have long been unaffected by the rise of TAVR; however, a modest decline in the number of SAVR procedures has been reported since 2years. ${ }^{12} 13$ Overall, the total 'AVR landscape' has significantly changed within the last $5-10$ years (figure 1 ), driven by new technologies and new clinical evidence.

Besides this shift in intervention mode, there is also new evidence regarding predictors of outcome in asymptomatic patients with severe aortic stenosis and on antithrombotic therapy in the SAVR and TAVR population. ${ }^{1415}$

This required an update of the ESC/ EACTS guidelines for the management of valvular heart disease. These guidelines on the management of valvular heart disease are joint guidelines between cardiologists and surgeons. ${ }^{15}$ It is absolutely essential that both specialties follow the same recommendations as they are treating the same patients.

This document aims to give an update and comprehensive overview on the position of TAVR in the latest $2017 \mathrm{ESC}$ /EACTS guidelines for the management of valvular heart disease.

\section{AORTIC STENOSIS-WHEN TO INTERVENE?}

When assessing a patient with aortic valve stenosis, some essential questions have to be asked and answered. How severe is the aortic stenosis? Does the patient have symptoms? Are the symptoms related to valvular disease? What are the patient's life expectancy and expected quality of life? Do the expected benefits of intervention outweigh its risks? Are local resources optimal for the planned intervention? What is the patient's preference?

Echocardiography is the key diagnostic tool. It confirms the presence of aortic valve 
2012

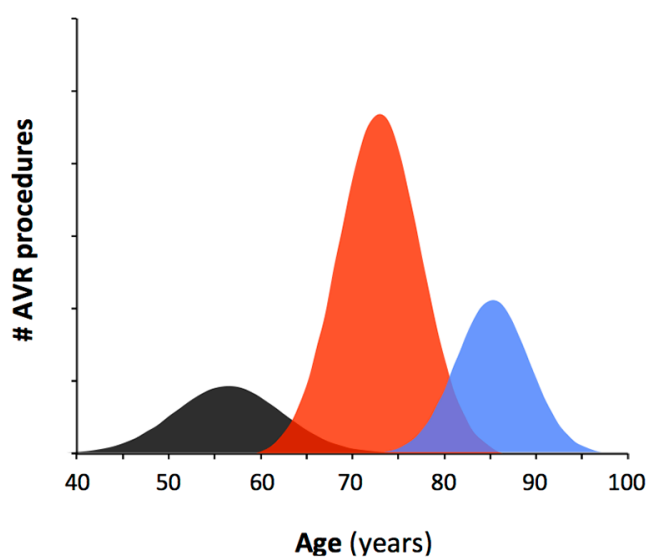

mech-SAVR
2017

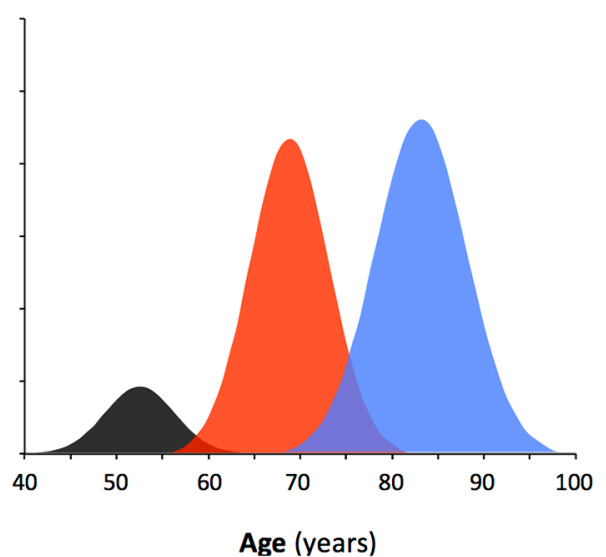

TAVR

Figure 1 Evolution of TAVR and SAVR in East Denmark between 2012 and 2017. AVR, aortic valve replacement; bio, biological; mech, mechanical; SAVR, surgical AVR; TAVR, transcatheter AVR.

stenosis; assesses the degree of valve calcification, left ventricular (LV)function and wall thickness and detects the presence of other associated valve disease or aortic pathology. Doppler echocardiography is the preferred technique for assessing the severity of the aortic valve stenosis.

In accordance with the 2012 ESC/EACTS guidelines, early therapy is strongly recommended in all symptomatic patients with severe high-gradient aortic stenosis (mean gradient $\geq 40 \mathrm{~mm} \mathrm{Hg}$ or peak velocity $\geq 4 \mathrm{~m} / \mathrm{s}$; class I, level of evidence (LoE) B). For this patient group, there is virtually no lower ejection fraction limit for intervention. The only exceptions are patients with severe comorbidities indicating a survival of $<1$ year and patients in whom severe comorbidities or their general condition at an advanced age make it unlikely that the intervention will improve quality of life or survival (class III, LoE C). ${ }^{415}$

The management of patients with low-gradient aortic stenosis is more challenging. In patients with low-flow, low-gradient aortic stenosis and reduced ejection fraction in whom the depressed ejection fraction is predominantly caused by excessive afterload, LV function usually improves after aortic valve replacement. ${ }^{16}{ }^{17}$ Intervention is indicated in symptomatic patients with severe low-flow, low-gradient $(<40 \mathrm{~mm} \mathrm{Hg})$ aortic stenosis with reduced ejection fraction, and evidence of flow (contractile) reserve excluding pseudosevere aortic stenosis (class I, LoE C).$^{15}$ Decision making about intervention should take into account the degree of valve calcification, the extent of coronary artery disease and the feasibility of concomitant or staged revascularisation, as well as the patient's comorbidities. The ability to identify patients with severe aortic stenosis in this subgroup by CT calcium scoring and the availability of TAVR have lowered the threshold to intervene. ${ }^{18-20}$ The new 2017 ESC/EACTS guidelines now also state that intervention 'should'—and not only 'may' (2012)—be considered in symptomatic patients with low-flow, low-gradient aortic stenosis and reduced ejection fraction without flow (contractile) reserve, particularly in those patients in whom CT calcium scoring confirms severe aortic stenosis (class IIa, LoE C).

Patients with low-flow, low-gradient aortic stenosis and preserved ejection fraction are the most difficult subgroup to decide on intervention or not. Data on their natural history and outcome after surgical or catheter intervention are scarce and remain controversial. ${ }^{21}{ }^{22} \mathrm{In}$ such cases, intervention should only be performed when symptoms are present and if comprehensive evaluation suggests significant valve obstruction. The strongest indication for intervention remains symptoms (either spontaneous or on exercise testing). ${ }^{15}$

Management of asymptomatic severe aortic stenosis remains controversial. The currently available studies do not provide convincing data to support the general recommendation of early SAVR - the decision to operate on asymptomatic patients requires careful weighing of the risks and benefits. In physically active patients with asymptomatic aortic stenosis, exercise testing is recommended for unmasking symptoms and for risk stratification. $^{2324}$

As mentioned in the ESC/EACTS guidelines, SAVR is indicated in asymptomatic patients with an impaired $\mathrm{LV}$ function $<50 \%$ not due to other causes and/or an abnormal exercise test showing symptoms on exercise related to aortic stenosis (class I, LoE C). Moreover, SAVR is indicated in asymptomatic patients with severe aortic stenosis undergoing coronary artery bypass graft (CABG) or surgery on the ascending aorta or another valve (class I, LoE C).$^{15}$

Interestingly, the updated guidelines now also state that SAVR should be considered in asymptomatic patients 
with normal ejection fraction if the surgical risk is low and one of the following findings is present: (1) very severe aortic stenosis defined by a peak velocity $>5.5 \mathrm{~m} / \mathrm{s}$, (2) severe valve calcification and a rate of peak velocity progression $\geq 0.3 \mathrm{~m} / \mathrm{s} /$ year, (3) markedly elevated brain natriuretic peptide (BNP) levels ( $>3$-fold normal range) confirmed by repeated measurements and (4) severe pulmonary hypertension (systolic pulmonary artery pressure at rest $>60 \mathrm{~mm} \mathrm{Hg}$ ) without other explanation. In patients without these predictive factors, watchful waiting appears safe and early surgery is unlikely to be beneficial. $^{15}$

For the time being, there is no evidence available showing a role for TAVR in the treatment of asymptomatic patients with severe aortic stenosis. However, studies are on their way to investigate the potential value of TAVR for this specific patient population.

\section{CHOICE OF INTERVENTION MODE-TAVR OR SAVR?}

The question which intervention mode is the preferred one to treat patients with severe, symptomatic aortic stenosis has been the subject of investigation in several important RCTs over the past decade. Based on the PARTNER IA/IB trials, the 2012 ESC/EACTS guidelines on the management of valvular heart disease formulated the following recommendations:

- TAVR is indicated in patients with severe symptomatic aortic stenosis who are not suitable for SAVR as assessed by a Heart Team and who are likely to gain improvement in their quality of life and to have a life expectancy of $>1$ year after consideration of their comorbidities (PARTNER IB; class I, LoE B) ${ }^{25}$

- TAVR should be considered in high-risk patients with severe symptomatic aortic stenosis who may still be suitable for surgery, but in whom TAVR is favoured based on the individual risk profile and anatomic suitability (PARTNER IA; class IIa, LoE B). ${ }^{5}$

Since 2012, outcomes of three other important clinical trials comparing TAVR and SAVR have been published. In the PARTNER-II, SURTAVI and NOTION trials, ${ }^{7-9}$ robust randomised data demonstrated the equivalence-and even net superiority when performed via transfemoral approach-of TAVR in intermediate-risk and lower-risk patients. This has set the stage for a further expansion of TAVR indications to encompass lower-risk patients in the updated 2017 ESC/EACTS guidelines on the management of valvular heart disease ${ }^{15}$ :

- TAVR is recommended in patients who are not suitable for SAVR as assessed by the Heart Team (class I, LoE B).

- In patients who are at increased surgical risk (Society of Thoracic Surgeons/STS or EuroSCORE II $\geq 4 \%$ or other risk factors not included in these scores such as frailty, porcelain aorta, sequelae of chest radiation), the decision between SAVR and TAVR should be made by the Heart Team according to the individual patient characteristics (see figure 2)-with TAVR being favoured in patients $>75$ years suitable for transfemoral access (class I, LoE B).

- In patients at low surgical risk (STS or EuroSCORE II $<4 \%$ and no other risk factors not included in these scores, such as frailty, porcelain aorta, sequelae of chest radiation), SAVR is recommended (class I, LoE B).

In the updated $2017 \mathrm{ESC} / \mathrm{EACTS}$ guidelines, patients are no longer divided into high risk, intermediate risk or low risk groups. Also the use of EuroSCORE I is no longer recommended to guide decision making. It is recommended to categorise patients into 'low surgical risk' or 'increased surgical risk', based on an STS or EuroSCORE II $<$ or $\geq 4 \%$, or other risk factors not included in these scores such as frailty, porcelain aorta, and sequelae of chest radiation.

The choice of intervention must be based on careful individual evaluation of technical suitability and weighing risks/benefits of each modality. In addition, the local expertise and outcome data for the given intervention must be taken into account. ${ }^{15}$

\section{Careful individual evaluation}

The choice of the intervention mode-SAVR or TAVRfor patients at increased surgical risk should take into account the cardiac and extracardiac characteristics of the patient as well as some important anatomical and technical aspects. Figure 2 provides aspects that should be considered for this individual decision. Importantly, current guidelines favour the use of transfemoral TAVR over surgery in elderly patients at increased surgical risk. In the two large studies on intermediate risk, the mean ages were 82 and 80 years and mean STS scores were $5.8 \%$ and $4.5 \% \cdot{ }^{89}$ Hence, the study results are valid for comparable patient groups.

\section{Frailty}

Besides specific organ comorbidities, there is growing interest in the assessment of frailty, an overall marker of impairment of functional, cognitive and nutritional status. ${ }^{26}{ }^{27}$ Frailty is associated with increased morbidity and mortality after surgery or TAVR. ${ }^{28} 29$ The assessment of frailty should not rely on a subjective approach, but rather on a combination of different objective estimates. Several tools are available for assessing frailty.

\section{Challenging subgroups}

There are a number of challenging subgroups with aortic valve disease that need special attention when considering TAVR. Data remain sparse for some specific patient cohorts excluded from key RCTs: low-flow, low-gradient aortic stenosis; patients with significant coronary artery or concomitant valve disease; aortic stenosis with severe left ventricular outflow tract calcification or previous valve surgery. Moreover, although recent registry data have shown good outcomes for TAVR treatment of bicuspid valves, unique anatomic challenges remain related to differences in annular size, patterns of leaflet calcification 


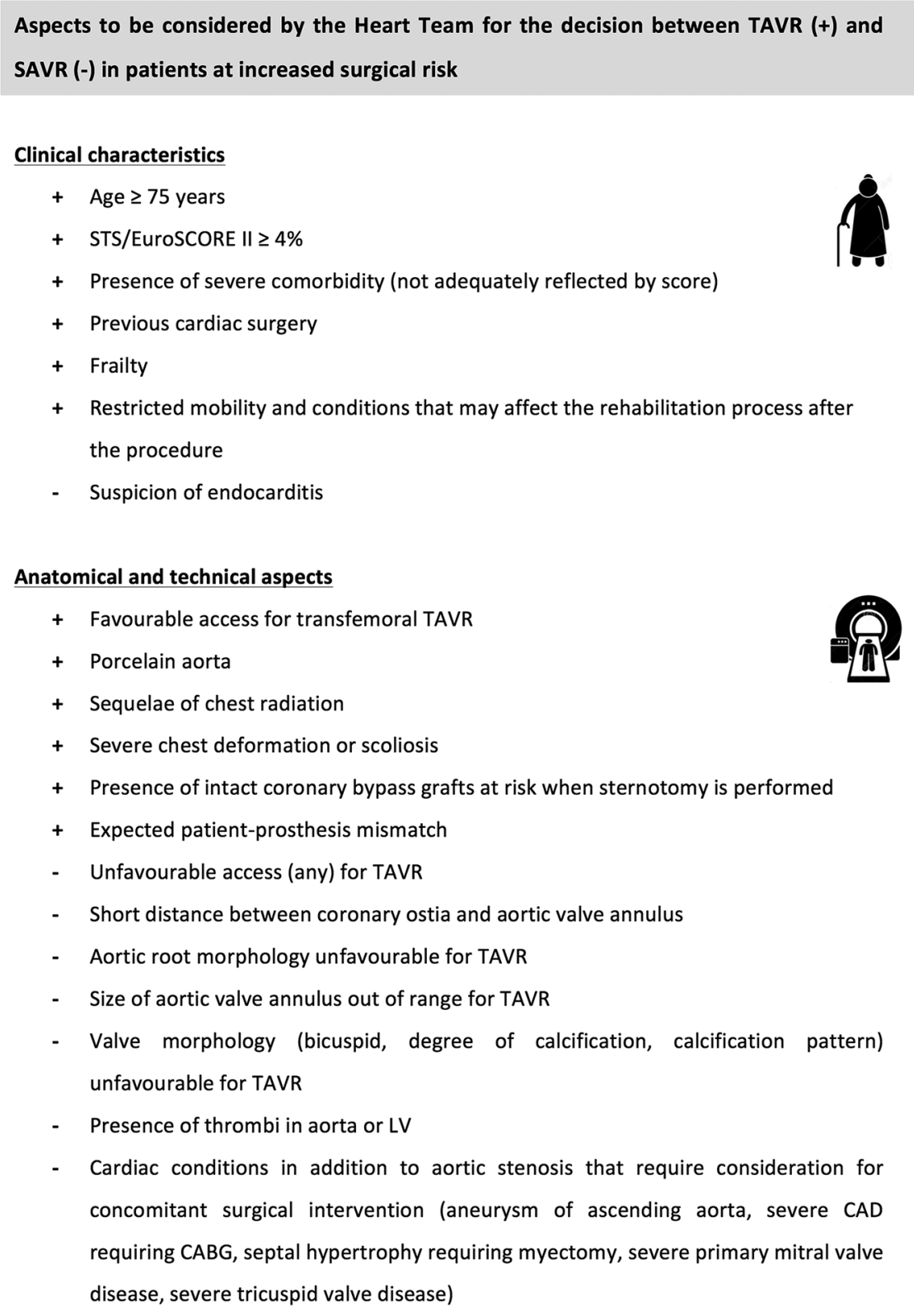

Figure 2 Aspects to be considered by the Heart Team for the decision between TAVR and SAVR in patients at increased surgical risk. (+) favours TAVR; (-) favours SAVR. Based on the recommendations formulated in the 2017 ESC/EACTS guidelines on the management of valvular heart disease. EACTS, European Association of CardioThoracic Surgery; ESC, European Society of Cardiology, SAVR, surgical aortic valve replacement; TAVR, transcatheter aortic valve replacement.

and orifice shape when compared with tri-leaflet valves. Different device types may also have different results in these complex patient subgroups. ${ }^{30-32}$

\section{Younger patients}

Data on TAVR are still very limited for patients below 75 years of age and for surgical low-risk patients, in whom SAVR remains the reference method. It has to be emphasised that younger patients differ with regard to anatomy (more bicuspid valves), which affects the results of TAVR (bicuspid valves were also in general excluded in clinical trials), and that long-term durability data for TAVR prosthetic valves are still lacking.

\section{Risks/benefits of SAVR and TAVR}

Overall, rates of vascular complications, paravalvular regurgitation (PVR) and pacemaker implantation are significantly higher for TAVR as compared with SAVR. In contrast, severe bleeding, kidney injury and new-onset atrial fibrillation are more frequent with surgery, whereas no difference has been observed in the rate of cerebrovascular events. ${ }^{7-9}$ Direct comparisons between valve types are now required to address potential discrepancies in the rates of vascular complication, PVR and pacemaker requirement. 
Vascular complications

Technological innovations continue the drive towards lower-profile delivery systems, and rates of major vascular complications have diminished considerably. In two recent high-risk registry studies examining outcomes after Evolut R implantation using a 14-Fr delivery system, major vascular complication rates were $5.3 \%$ and $7.5 \%$, respectively. ${ }^{3334}$ Nevertheless, there are large variations in sheath requirements for currently available TAVR systems (14-22 Fr), and vascular complications remain an important source of immediate-term and long-term comorbidity.

\section{Paravalvular regurgitation}

Moderate or severe PVR was initially reported in 10\%-15\% of patients treated with TAVR and has been associated with increased mortality. ${ }^{35}$ However, more accurate sizing of the aortic annulus based on multislice computed tomography (MSCT) imaging-instead of transoesophageal echocardiography (TOE) imaging-resulted in better PVR outcomes over the past few years. In addition, newer generation transcatheter heart valves have an outer skirt or adaptive seal, and some systems are even repositionable to optimise the implantation position. These improvements have resulted in moderate-to-severe PVR rates $<5 \%$ in the latest TAVR studies ${ }^{36}{ }^{37}$ - still, further improvements and reduction in the PVR rates will be required for lower risk cohorts with longer life expectancy.

\section{Pacemaker implantation}

Due to the proximity between the transcatheter valve frame extending into the left ventricular outflow tract and the conduction system, heart block with need for permanent pacemaker implantation has been frequent after TAVR. Although new permanent pacemaker implantation adds to the risk of procedural complications and overall cost, it protects against unexpected death, probably due to the inherent risk of complete heart block among patients with severe aortic stenosis. Longer-term follow-up studies will have to investigate the impact of this permanent pacemaker implantation/ use on left ventricular function, risk for device-related infection and quality of life. The appreciation of the importance of higher prosthesis implantation as well as introduction of repositionable TAVR systems has lowered the need for permanent pacemaker to $<15 \%$ for most systems. ${ }^{38} 39$

\section{TAVR_DIAGNOSTIC WORK-UP AND PROCEDURE}

Since the start of TAVR now more than 10 years ago, many aspects have changed-both in the diagnostic work-up as well as in the performance of the TAVR procedure itself. While TAVR was only a treatment option for extreme and high surgical risk patients in the early days, the indication for TAVR has now expanded to lower-risk patients. This evolution has gone hand-in-hand with a standardisation and simplification of the entire TAVR work flow.

The diagnostic work-up before TAVR was in the early days mainly limited to a TOE and coronary angiography (CAG) including an aortography and angiography of the iliofemoral arteries. In the updated 2017 ESC/ EACTS guidelines, it is now clearly stated that MSCT is the preferred imaging tool to assess the anatomy and dimensions of the aortic root, size and shape of the aortic valve annulus, its distance to the coronary ostia, the distribution of calcifications and the number of aortic valve cusps. It is essential to evaluate the feasibility of the various access routes, as this provides information on minimal luminal diameters, atherosclerotic plaque burden, the presence of aneurysms or thrombi, vessel tortuosity and thoracic and left ventricular apex anatomy. ${ }^{15}$ Cardiac magnetic resonance-as an alternative technique-is, in this context, inferior to MSCT with regard to assessment of inner vessel dimensions and calcifications. The use of TOE to determine aortic annulus dimensions can still be relevant in patients who cannot undergo a contrast-enhanced MSCT (eg, patients with severely reduced renal function)however, it is important to realise that TOE remains more operator-dependent and image quality-dependent than MSCT. Still, TOE can still be an important tool for monitoring the procedure and evaluating the results, especially if complications occur. ${ }^{40}$

Concerning the need for a CAG before TAVR, the 2017 ESC/EACTS guidelines mention that a CAG is recommended before valve surgery in all men $>40$ years of age and postmenopausal women ${ }^{15}$ - in accordance, a diagnostic CAG should be considered for all patients referred to TAVR. Combination of percutaneous coronary intervention (PCI) and TAVR has been shown to be feasible but requires more data before a firm recommendation can be made. The chronology of interventions should be the subject of individualised discussion based on the patient's clinical condition, extent of coronary artery disease and myocardium at risk. It is a new recommendation in the 2017 guidelines that PCI should be considered in patients with a primary indication to undergo TAVR and coronary artery diameter stenosis $>70 \%$ in proximal segments (class IIa, LoE C). It is increasingly accepted to leave borderline coronary artery stenosis and/or stenosis located in more distal coronary segments untreatedonly in case of obvious pre-TAVR angina symptoms, treatment of these lesions could be preferred. ${ }^{15}$

In order to standardise the TAVR procedure, it is recommended that these procedures are performed in dedicated heart valve centres. The main purpose of heart valve centres as centres of excellence in the treatment of valvular heart disease is to deliver better quality of care. This is achieved through greater volumes associated with specialisation of training, continuing education and clinical interest. Techniques with a steep learning curve may be performed with better results in hospitals with high volumes and more experience. ${ }^{41}$ 
The updated 2017 ESC/EACTS guidelines state that aortic valve interventions should only be performed in centres with both departments of cardiology and cardiac surgery on-site and with structured collaboration between the two, including a Heart Team (class I, LoE C). Expertise in interventional and surgical management of vascular diseases and complications must be available. In addition, it is recommended to record data on performance and patient outcome at the level of the given heart valve centre and to participate in national or ESC/ EACTS registries. ${ }^{15}$

In accordance with the standardisation of the TAVR procedure-there has been a significant simplification of TAVR, especially within the last few years. The availability of new transcatheter heart valves with smaller insertion profiles has resulted in an increasing number of TAVR procedures performed by transfemoral approach. Even to such an extent that in most large-volume centres, the transfemoral approach accounts for more than $90 \%$ of all TAVR procedures. This evolution has been accompanied by—or has (partially) resulted into- a switch from general anaesthesia for all TAVR procedures to mainly TAVR procedures in local anaesthesia with or without conscious sedation. ${ }^{42}{ }^{43}$ In many centres in Europe, general anaesthesia is now only reserved for TAVR procedures which need an alternative access, for example, subclavian, transapical, direct aortic or transcaval approach. Because of this evolution, many other periprocedural aspects have also been simplified: no more use of a central venous line, bladder catheter, stay at intensive care unit -also referred to as 'minimalistic TAVR'. ${ }^{43-45}$ All together, this has resulted in significantly shorter lengths of hospitalisation with often discharge $24-48$ hours after TAVR. In addition, this 'fast track' TAVR also results in a significant cost reduction, which is increasingly important in current healthcare. ${ }^{456}$

\section{POST-TAVR MANAGEMENT}

Patients who receive a surgical or transcatheter heart valve should have a transthoracic echocardiography before discharge. Echocardiography should be routinely performed within 30-90 days to establish baseline valve function, at 1 year after AVR and then annually thereafter. Important parameters to follow are (the temporal evolution of) the transvalvular gradient, the degree of $\mathrm{PVR} /$ central regurgitation and the overall left ventricular function.

Regarding antithrombotic treatment following TAVR, the new 2017 ESC/EACTS guidelines state that dual antiplatelet therapy should be considered for 3-6months after TAVR, followed by lifelong single therapy in patients who do not need oral anticoagulation for other reasons (class IIa, LoE C). Single antiplatelet therapy may be considered after TAVR in the case of bleeding risk (class IIb, LoE C). In patients with TAVR with atrial fibrillation, novel oral anticoagulants should be considered as an alternative to vitamin $\mathrm{K}$ antagonists (VKA) after the third month of valve implantation (class IIa, LoE C). ${ }^{15}$

Recently, subclinical leaflet thrombosis has been reported to occur in bioprosthetic aortic valves, more commonly in transcatheter than in surgical valves. This subclinical thrombosis of bioprosthesis may be more frequent when assessed by cardiac CT and associated with a moderate increase in transprosthetic gradients but the clinical consequences are unknown. ${ }^{47}$ It has been speculated that this phenomenon has importance with regard to increased risk of thromboembolic (neurological) events and/or reduced valve durability. Anticoagulation, but not dual antiplatelet therapy, is effective in prevention or treatment of subclinical leaflet thrombosis. ${ }^{47}$ Anticoagulant using a VKA and/or unfractionated heparin is the first-line treatment of bioprosthetic valve thrombosis. Despite excellent outcomes after TAVR with the new-generation valves, prevention and treatment of subclinical leaflet thrombosis might offer a potential opportunity for further improvement in clinical outcomes. Two ongoing randomised controlled trials (GALILEO, ATLANTIS) are specifically investigating which antithrombotic strategy might be best following TAVR.

Finally, in case of bioprosthetic valve failure with severe restenosis or aortic regurgitation (eg, degenerative, post-endocarditis), transcatheter valve-in-valve implantation is an option in this higher risk patient population.

\section{CONCLUSION}

Since the 2012 ESC/EACTS guidelines on the management of valvular heart disease, a large amount of new data have accumulated, in particular in the field of TAVR. Indications for TAVR have expanded as there is new evidence for lower-risk populations. This required an update of the recommendations in the new 2017 guidelines. Broadly speaking, patients at higher risk for surgery should receive TAVR-especially if they can be treated by transfemoral approach-and those at low risk-in particular younger patients-should undergo SAVR. The choice of SAVR or TAVR should not simply be based on a risk score or age. The Heart Team must weigh the risks and benefits of both procedures, particularly in the intermediate risk situation. Discussion should include age, comorbidities, anatomy and outcomes of the centre for surgery and transcatheter intervention.

Funding This research received no specific grant from any funding agency in the public, commercial or not-for-profit sectors.

Competing interests None declared.

Provenance and peer review Not commissioned; externally peer reviewed.

Open Access This is an Open Access article distributed in accordance with the Creative Commons Attribution Non Commercial (CC BY-NC 4.0) license, which permits others to distribute, remix, adapt, build upon this work non-commercially, and license their derivative works on different terms, provided the original work is properly cited and the use is non-commercial. See: http://creativecommons.org/ licenses/by-nc/4.0/ 
c) Article author(s) (or their employer(s) unless otherwise stated in the text of the article) 2018. All rights reserved. No commercial use is permitted unless otherwise expressly granted.

\section{REFERENCES}

1. Osnabrugge RL, Mylotte D, Head SJ, et al. Aortic stenosis in the elderly: disease prevalence and number of candidates for transcatheter aortic valve replacement: a meta-analysis and modeling study. J Am Coll Cardiol 2013;62:1002-12.

2. Coffey S, Cox B, Williams A. The prevalence, incidence, progression, and risks of aortic valve sclerosis: a systemic review and metaanalysis. J Am Coll Cardiol 2013;63:2852-61.

3. Mylotte D, Osnabrugge RLJ, Windecker S, et al. Transcatheter aortic valve replacement in Europe: adoption trends and factors influencing device utilization. J Am Coll Cardiol 2013;62:210-9.

4. Vahanian A, Alfieri O, Andreotti F, et al. Guidelines on the management of valvular heart disease (version 2012): the joint tasks force on management of valvular heart disease of the European Society of Cardiology (ESC) and the European Association for Cardio-Thoracic Surgery (EACTS). Eur Heart J 2012;33:2451-96.

5. Smith $\mathrm{CR}$, Leon MB, Mack MJ, et al. for the PARTNER Trial investigators. Transcatheter versus surgical aortic-valve replacement in high-risk patients. N Engl J Med 2011;362:2187-98.

6. Adams DH, Popma JJ, Reardon MJ, et al. for the U.S. CoreValve clinical investigators. Transcatheter aortic-valve replacement with a self-expanding prosthesis. N Engl J Med 2014;370:1790-8.

7. Thyregod HG, Steinbrüchel DA, Ihlemann N, et al. Transcatheter versus surgical aortic valve replacement in patients with severe aortic valve stenosis: 1-year results from the all-comers notion randomized clinical trial. J Am Coll Cardiol 2015;65:2184-94.

8. Leon MB, Smith CR, Mack MJ, et al. for the PARTNER 2 Investigators. Transcatheter or surgical aortic valve replacement in intermediate-risk patients. N Engl J Med 2016;374:1609-20.

9. Reardon MJ, Mieghem NM, Popma JJ, et al. for the SURTAV Investigators. Surgical or transcatheter aortic-valve replacement in intermediate-risk patients. N Engl J Med 2017;376:1321-31.

10. Rosato S, Santini F, Barbanti M, et al. on behalf of the OBSERVANT research group. Transcatheter aortic valve implantation compared with surgical aortic valve replacement in low-risk patients. Circ Cardiovasc Interv 2016;9:e003326.

11. Arora S, Strassle PD, Ramm CJ, et al. Transcatheter versus surgical aortic valve replacement in patients with lower surgical risk scores: a systematic review and meta-analysis of early outcomes. Heart Lung Circ 2017;26:840-5.

12. Eggebrecht $\mathrm{H}$, Mehta $\mathrm{RH}$. Transcatheter aortic valve implantation (TAVI) in Germany 2008-2014: on its way to standard therapy for aortic valve stenosis in the elderly? Eurolntervention 2016;11:1029-33.

13. Hawkins RB, Downs EA, Johnston LE, et al. for investigators for the Virginia cardiac services quality initiative. Impact of transcatheter technology on surgical aortic valve replacement volume, outcomes, and cost. Ann Thorac Surg 2017;103:1815-23.

14. Vavuranakis M, Siasos G, Zografos T, et al. Dual or single antiplatelet therapy after transcatheter aortic valve implantation? a systematic review and meta-analysis. Curr Pharm Des 2016;22:4596-603.

15. Baumgartner $\mathrm{H}$, Falk V, Bax JJ, et al. ESC/EACTS guidelines for the management of valvular heart disease: the task force for the management of valvular heart disease of the European Society of Cardiology (ESC) and European Association for Cardio-Thoracic Surgery (EACTS). Eur Heart J 2017;2017:2739-86.

16. Monin JL, Quéré JP, Monchi M, et al. Low-gradient aortic stenosis: operative risk stratification and predictors for long-term outcome: a multicenter study using dobutamine stress hemodynamics. Circulation 2003;108:319-24.

17. Tribouilloy C, Lévy F, Rusinaru D, et al. Outcome after aortic valve replacement for low-flow/low-gradient aortic stenosis without contractile reserve on dobutamine stress echocardiography. J Am Coll Cardiol 2009;53:1865-73.

18. Cueff C, Serfaty JM, Cimadevilla C, et al. Measurement of aortic valve calcification using multislice computed tomography: correlation with haemodynamic severity of aortic stenosis and clinical implication for patients with low ejection fraction. Heart 2011;97:721-6.

19. Clavel MA, Messika-Zeitoun D, Pibarot P, et al. The complex nature of discordant severe calcified aortic valve disease grading: new insights from combined Doppler echocardiographic and computed tomographic study. J Am Coll Cardiol 2013;62:2329-38.

20. Clavel MA, Pibarot $P$, Messika-Zeitoun $D$, et al. Impact of aortic valve calcification, as measured by MDCT, on survival in patients with aortic stenosis: results of an international registry study. J Am Coll Cardiol 2014;64:1202-13.

21. Herrmann HC, Pibarot P, Hueter I, et al. Predictors of mortality and outcomes of therapy in low-flow severe aortic stenosis: a placement of aortic transcatheter valves (PARTNER) trial analysis. Circulation 2013;127:2316-26.

22. Ozkan A, Hachamovitch R, Kapadia SR, et al. Impact of aortic valve replacement on outcome of symptomatic patients with severe aortic stenosis with low gradient and preserved left ventricular ejection fraction. Circulation 2013;128:622-31.

23. Rafique AM, Biner S, Ray I, et al. Meta-analysis of prognostic value of stress testing in patients with asymptomatic severe aortic stenosis. Am J Cardiol 2009;104:972-7.

24. Maréchaux S, Hachicha Z, Bellouin A, et al. Usefulness of exercise-stress echocardiography for risk stratification of true asymptomatic patients with aortic valve stenosis. Eur Heart $J$ 2010;31:1390-7.

25. Leon MB, Smith CR, Mack M, et al. for the PARTNER trial investigations. Transcatheter aortic valve implantation for aortic stenosis in patients who cannot undergo surgery. N Engl J Med 2010;363:1597-607

26. Cockburn J, Singh MS, Rafi NHM, et al. Poor mobility predicts adverse outcome better than other frailty indices in patients undergoing transcatheter aortic valve implantation. Cath Cardiovasc Interv 2015;86:1271-7.

27. Assmann P, Kievit P, van der Wulp K, et al. Frailty is associated with delirium and mortality after transcatheter aortic valve implantation. Open Heart 2016;3:e000478.

28. Puls M, Sobisiak B, Bleckmann A, et al. Impact of frailty on shortand long-term morbidity and mortality after transcatheter aortic valve implantation: risk assessment by Katz index of activities of daily living. Eurolntervention 2014;10:609-19.

29. Green P, Arnold SV, Cohen DJ, et al. Relation of frailty to outcomes after transcatheter aortic valve replacement (from the PARTNER trial). Am J Cardiol 2015;116:264-9.

30. Bauer T, Linke A, Sievert $\mathrm{H}$, et al. Comparison of the effectiveness of transcatheter aortic valve implantation in patients with stenotic bicuspid versus tricuspid aortic valves (from the German TAVI Registry). Am J Cardiol 2014;113:518-21.

31. Phan K, Wong S, Phan S, et al. Transcatheter aortic valve implantation (tavi) in patients with bicuspid aortic valve stenosis--systematic review and meta-analysis. Heart Lung Circ 2015;24:649-59.

32. Mylotte D, Lefevre T, Søndergaard L, et al. Transcatheter aortic valve replacement in bicuspid aortic valve disease. J Am Coll Cardiol 2014;64:2330-9.

33. Kalra SS, Firoozi S, Yeh J, et al. Initial experience of a secondgeneration self-expanding transcatheter aortic valve: the uk \& ireland evolut $r$ implanters' registry. JACC Cardiovasc Interv 2017;10:276-82.

34. Popma JJ, Reardon MJ, Khabbaz K, et al. Early clinical outcomes after transcatheter aortic valve replacement using a novel selfexpanding bioprosthesis in patients with severe aortic stenosis who are suboptimal for surgery: results of the evolut $r$ u.s. study. JACC Cardiovasc Interv 2017;10:268-75.

35. Van Belle E, Juthier F, Susen S, et al. Postprocedural aortic regurgitation in balloon-expandable and self-expandable transcatheter aortic valve replacement procedures: analysis of predictors and impact on long-term mortality: insights from the FRANCE2 Registry. Circulation 2014;129:1415-27.

36. Arai $T$, Lefèvre $T$, Hovasse $T$, et al. Comparison of Edwards SAPIEN 3 versus SAPIEN XT in transfemoral transcatheter aortic valve implantation: Difference of valve selection in the real world. $J$ Cardiol 2017;69:565-9.

37. Meredith IT, Walters DL, Dumonteil N, et al. 1-Year Outcomes With the Fully Repositionable and Retrievable Lotus Transcatheter Aortic Replacement Valve in 120 High-Risk Surgical Patients With Severe Aortic Stenosis: Results of the REPRISE II Study. JACC Cardiovasc Interv 2016;9:376-84.

38. Tummala R, Banerjee K, Sankaramangalam K, et al. Clinical and procedure outcomes with SAPIEN3 versus the SAPIEN XT prosthetic valves in transcatheter aortic valve replacement: a systematic review and meta-analysis. Cath Cardiovasc Interv 2017;00:1-10.

39. Sorajia P, Kodali S, Reardon MJ, et al. Outcomes for the commercial use of self-expanding prostheses in transcatheter aortic valve replacement: a report from the sts/acc tvt registry. JACC Cardiovasc Interv 2017;10:2090-8.

40. Zamorano J, Gonçalves A, Lancellotti P, et al. The use of imaging in new transcatheter interventions: an EACVI review paper. Eur Heart $J$ Cardiovasc Imaging 2016;17:835-835af. 
41. Badheka AO, Patel NJ, Panaich SS, et al. Effect of hospital volume on outcomes of transcatheter aortic valve implantation. Am J Cardiol 2015;116:587-94.

42. Durand E, Borz B, Godin M, et al. Transfemoral aortic valve replacement with the Edwards SAPIEN and Edwards SAPIEN XT prosthesis using exclusively local anesthesia and fluoroscopic guidance: feasibility and 30-day outcomes. JACC Cardiovasc Interv 2012;5:461-7.

43. Sawaya FJ, Lefèvre T, Spaziano $\mathrm{M}$, et al. Transfemoral transcatheter aortic valve implantation: how minimalistic can we become? J Interv Cardiol 2016;29:628-31

44. Babaliaros V, Devireddy C, Lerakis S, et al. Comparison of transfemoral transcatheter aortic valve replacement performed in the catheterization laboratory (minimalist approach) versus hybrid operating room (standard approach): outcomes and cost analysis. JACC Cardiovasc Interv 2014;7:898-904.

45. Alkhalil A, Lamba H, Deo S, et al. Safety of shorter length of hospital stay for patients undergoing minimalist transcatheter aortic valve replacement. Cath Cardiovasc Interv 2017;00:1-9.

46. Marcantuono R, Gutsche J, Burke-Julien M, et al. Rationale, development, implementation, and initial results of a fast track protocol for transfemoral transcatheter aortic valve replacement (TAVR). Cath Cardiovasc Interv 2015;85:648-54.

47. Chakravarty T, Søndergaard L, Friedman J, et al. on behalf of the RESOLVE and SAVORY investigators. Subclinical leaflet thrombosis in surgical and transcatheter bioprosthetic aortic valves: an observational study. Lancet 2017;389:2383-92. 